\title{
Solitons and Jacobi Elliptic Function Solutions to the Complex Ginzburg-Landau Equation
}

\author{
Kamyar Hosseini 1*, Mohammad Mirzazadeh ${ }^{2}$, M. S. Osman ${ }^{3,4}$, Maysaa Al Qurashi ${ }^{5}$ and \\ Dumitru Baleanu ${ }^{6,7}$ \\ ${ }^{1}$ Department of Mathematics, Rasht Branch, Islamic Azad University, Rasht, Iran, ${ }^{2}$ Department of Engineering Sciences, \\ Faculty of Technology and Engineering, East of Guilan, University of Guilan, Rudsar-Vajargah, Iran, ${ }^{3}$ Department of \\ Mathematics, Faculty of Science, Cairo University, Giza, Egypt, ${ }^{4}$ Department of Mathematics, Faculty of Applied Science, \\ Umm Alqura University, Makkah, Saudi Arabia, ${ }^{5}$ Department of Mathematics, King Saud University, Riyadh, Saudi Arabia, \\ ${ }^{6}$ Department of Mathematics, Faculty of Arts and Sciences, Cankaya University, Ankara, Turkey, ${ }^{7}$ Institute of Space \\ Sciences, Magurele-Bucharest, Romania
}

The complex Ginzburg-Landau (CGL) equation which describes the soliton propagation in the presence of the detuning factor is firstly considered; then its solitons as well as Jacobi elliptic function solutions are obtained systematically using a modified Jacobi elliptic expansion method. In special cases, several dark and bright soliton solutions to the CGL equation are retrieved when the modulus of ellipticity approaches unity. The results presented in the current work can help to complete previous studies on the complex Ginzburg-Landau equation.

OPEN ACCESS

Edited by:

Grienggrai Rajchakit,

Maejo University, Thailand

Reviewed by:

Yilun Shang,

Northumbria University,

United Kingdom

Alexander I. Nesterov,

Universidad de Guadalajara, Mexico

*Correspondence:

Kamyar Hossein

kamyar_hosseini@yahoo.com

Specialty section:

This article was submitted to

Mathematical Physics,

a section of the journal

Frontiers in Physics

Received: 23 April 2020

Accepted: 25 May 2020

Published: 30 June 2020

Citation:

Hosseini K, Mirzazadeh M,

Osman MS, Al Qurashi M and

Baleanu D (2020) Solitons and Jacobi

Elliptic Function Solutions to the Complex Ginzburg-Landau Equation.

Front. Phys. 8:225.

doi: 10.3389/fphy.2020.00225
Keywords: complex Ginzburg-Landau equation, detuning factor, modified Jacobi elliptic expansion method, solitons, Jacobi elliptic function solutions

\section{INTRODUCTION}

One of the very hot topics in many of today's research is the search for exact solutions of non-linear partial differential (NLPD) equations. As it is known, exact solutions of NLPD equations play a significant role in a wide variety of applied sciences and provide meaningful information about physical phenomena modeled by non-linear partial differential equations. Fortunately, with the development of symbolic computation packages, a variety of systematic methods [1-34] (Hosseini et al., under review) have been proposed to obtain exact solutions of NLPD equations. Many of these methods are based on considering the solution as a finite series in terms of the solutions of well-known ordinary differential equations such as Bernoulli, Riccati, and Jacobi equations. Among these methods, the modified Jacobi elliptic expansion (MJEE) method [21-27] has achieved a great deal of attention. For instance, El-Sheikh et al. [25] extracted elliptic function solutions of nonlinear Boussinesq-like equations using the MJEE method. Hosseini et al. [26] applied the MJEE method to find optical solitons of the Fokas-Lenells equation. Hosseini et al. [27] also obtained exact solutions of a $(3+1)$-dimensional resonant non-linear Schrödinger equation through the MJEE method. Such results encouraged the authors of the present article to employ the modified Jacobi elliptic expansion method to derive complex wave structures of the following complex Ginzburg-Landau equation [28-34] (Hosseini et al., under review)

$$
\begin{aligned}
& i \frac{\partial u(x, t)}{\partial t}+\alpha_{1} \frac{\partial^{2} u(x, t)}{\partial x^{2}}+\alpha_{2}|u(x, t)|^{2} u(x, t)-\frac{\alpha_{3}}{|u(x, t)|^{2} u^{*}(x, t)}\left(2|u(x, t)|^{2} \frac{\partial^{2}|u(x, t)|^{2}}{\partial x^{2}}\right. \\
& \left.-\left(\frac{\partial|u(x, t)|^{2}}{\partial x}\right)^{2}\right)-\alpha_{4} u(x, t)=0,
\end{aligned}
$$


which describes the soliton propagation in the presence of the detuning factor. In the CGL Equation (1), $\alpha_{1}$ and $\alpha_{2}$ are the coefficients of the group velocity dispersion and the Kerr law non-linearity while $\alpha_{3}$ and $\alpha_{4}$ denote the coefficients of the perturbation effects, especially $\alpha_{4}$ comes from the detuning effect. Recently, the main subject of a lot of studies has been focused on the complex Ginzburg-Landau equation and its exact solutions. Osman et al. [30] reported several complex wave structures to the CGL equation using a modified auxiliary equation method. In another paper, Abdou et al. [31] obtained dark-singular combo solitons of the CGL equation through the extended Jacobi elliptic expansion method. Solitons of the CGL equation were derived by Arshed in [32] with the help of the $\exp (-\varphi(\epsilon))$-expansion method. Rezazadeh [33] listed a series of soliton solutions to the CGL equation using a new extended direct algebraic method, and finally, Arnous et al. [34] employed the modified simple equation method to generate optical solitons of the CGL equation. This paper is arranged as follows: In Section the modified Jacobi elliptic expansion method and its fundamental, the modified Jacobi elliptic expansion method and its fundamental is reviewed. In Section complex Ginzburg-Landau equation and its exact solutions, exact solutions of the CGL equation are given by applying the MJEE method. Section conclusion is devoted to summarizing the conclusions.

\section{THE MODIFIED JACOBI ELLIPTIC EXPANSION METHOD AND ITS FUNDAMENTAL}

To explain the basic ideas of the modified Jacobi elliptic expansion method, we consider the following NLPD equation

$P\left(u, u_{x}, u_{t}, u_{x x}, u_{x t}, u_{t t}, \ldots\right)=0, \quad P$ is a function, $u$ is an unknown

and suppose that it can be converted to an ODE as follows

$$
O\left(U, U^{\prime}, U^{\prime \prime}, \ldots\right)=0, \quad{ }^{\prime}=\frac{d}{d \xi},
$$

through considering the transformation $u(x, t)=$ $U(\xi) e^{i(-\kappa x+\omega t+\theta)}$ which $\xi=x-v t$.

Now, assume that the non-trivial solution of Equation (3) can be expressed as

$$
\begin{aligned}
& U(\xi)=c_{0}+\sum_{i=1}^{N}\left(\frac{J(\xi)}{1+J^{2}(\xi)}\right)^{i-1}\left(c_{i} \frac{J(\xi)}{1+J^{2}(\xi)}+d_{i} \frac{1-J^{2}(\xi)}{1+J^{2}(\xi)}\right) \\
& c_{N} \text { or } d_{N} \neq 0
\end{aligned}
$$

where $c_{0}, c_{i}$, and $d_{i}(1 \leq i \leq N)$ are constants to be found later, $N$ is the balance number, and $J(\xi)$ is a known function satisfying the following Jacobi elliptic equation

$$
\left(J^{\prime}(\xi)\right)^{2}=D+E J^{2}(\xi)+F J^{4}(\xi) .
$$

The Jacobi elliptic Equation (5) admits the following exact solutions (See Table 1):
TABLE 1 | Jacobi elliptic function solutions of Equation (5).

\begin{tabular}{ccccc}
\hline No. & $\boldsymbol{D}$ & $\boldsymbol{E}$ & $\boldsymbol{F}$ & $\boldsymbol{J}(\xi)$ \\
\hline 1 & 1 & $-\left(m^{2}+1\right)$ & $m^{2}$ & $\operatorname{sn}(\xi)$ \\
2 & $1-m^{2}$ & $2 m^{2}-1$ & $-m^{2}$ & $\operatorname{cn}(\xi)$ \\
3 & $m^{2}$ & $-\left(m^{2}+1\right)$ & 1 & $n s(\xi)$ \\
4 & $-m^{2}$ & $2 m^{2}-1$ & $1-m^{2}$ & $n c(\xi)$ \\
\hline
\end{tabular}

By substituting the solution (4) along with the Jacobi elliptic Equation (5) into Equation (3) and performing a series of calculations, we arrive at a set of non-linear algebraic equations whose solution yields exact solutions of the NLPD Equation (2).

The Jacobi elliptic functions include some interesting properties that are reviewed below [35]:

- $s n^{2}(\xi)+c n^{2}(\xi)=1$.

- $s n(\xi)=s n(\xi, m) \rightarrow \tanh (\xi)$ when $m \rightarrow 1$.

- $n s(\xi)=(\operatorname{sn}(\xi, m))^{-1} \rightarrow \operatorname{coth}(\xi)$ when $m \rightarrow 1$.

\section{COMPLEX GINZBURG-LANDAU EQUATION AND ITS EXACT SOLUTIONS}

To gain exact solutions of the CGL equation, we first apply a complex transformation in the form below

$$
u(x, t)=U(\xi) e^{i(-\kappa x+\omega t+\theta)}, \quad \xi=x-v t,
$$

where $v$ is the velocity of the soliton whereas $\kappa, \omega$, and $\theta$ are the wave number, the frequency, and the phase constant, respectively. Such a complex transformation results in

$$
\left(-\alpha_{1}+4 \alpha_{3}\right) \frac{d^{2} U(\xi)}{d \xi^{2}}+\left(\kappa^{2} \alpha_{1}+\omega+\alpha_{4}\right) U(\xi)-\alpha_{2} U^{3}(\xi)=0
$$

where the velocity of the soliton is

$$
v=-2 \kappa \alpha_{1} .
$$

Now, based on the Jacobi elliptic expansion method and the balance number $N=1$, the solution of Equation (6) is considered as follows

$$
U(\xi)=c_{0}+c_{1} \frac{J(\xi)}{1+J^{2}(\xi)}+c_{2} \frac{1-J^{2}(\xi)}{1+J^{2}(\xi)}, \quad c_{2}=d_{1},
$$

where $c_{0}, c_{1}$, and $c_{2}$ are constants to be found later. By inserting the above solution along with the Jacobi elliptic Equation (5) into Equation (6) and performing a series of calculations, we will get a set of non-linear algebraic equations as

$$
\begin{aligned}
& -\kappa^{2} \alpha_{1} c_{0}-\kappa^{2} \alpha_{1} c_{2}+\alpha_{2} c_{0}{ }^{3}+3 \alpha_{2} c_{0}{ }^{2} c_{2}+3 \alpha_{2} c_{0} c_{2}^{2}+\alpha_{2} c_{2}{ }^{3} \\
& -4 D \alpha_{1} c_{2}+16 D \alpha_{3} c_{2}-\omega c_{0}-\omega c_{2}-\alpha_{4} c_{0}-\alpha_{4} c_{2}=0 \\
& -\kappa^{2} \alpha_{1} c_{1}+3 \alpha_{2} c_{0}^{2} c_{1}+6 \alpha_{2} c_{0} c_{1} c_{2}+3 \alpha_{2} c_{1} c_{2}^{2}-6 D \alpha_{1} c_{1} \\
& +24 D \alpha_{3} c_{1}+E \alpha_{1} c_{1}-4 E \alpha_{3} c_{1}-\omega c_{1}-\alpha_{4} c_{1}=0 \\
& -3 \kappa^{2} \alpha_{1} c_{0}-\kappa^{2} \alpha_{1} c_{2}+3 \alpha_{2} c_{0}{ }^{3}+3 \alpha_{2} c_{0}^{2} c_{2}+3 \alpha_{2} c_{0} c_{1}^{2}
\end{aligned}
$$



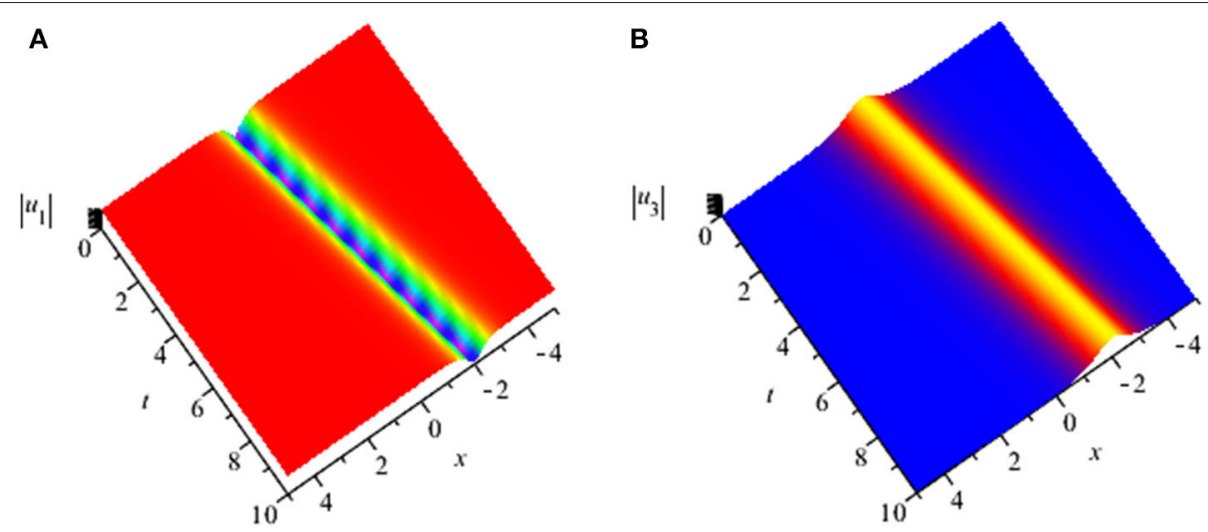

FIGURE 1 | (A) The 3-dimensional graph of $\left|u_{1}(x, t)\right|$ for $\alpha_{1}=1, \alpha_{2}=1, \alpha_{3}=1, \alpha_{4}=1, \kappa=0.1$, and $\theta=0$; (B) The 3-dimensional graph of $\left|u_{3}(x, t)\right|$ for $\alpha_{1}=1$, $\alpha_{2}=1, \alpha_{3}=1, \alpha_{4}=1, \kappa=0.1$, and $\theta=0$.

$$
\begin{aligned}
& -3 \alpha_{2} c_{0} c_{2}^{2}+3 \alpha_{2} c_{1}^{2} c_{2}-3 \alpha_{2} c_{2}^{3}+12 D \alpha_{1} c_{2}-48 D \alpha_{3} c_{2} \\
& -8 E \alpha_{1} c_{2}+32 E \alpha_{3} c_{2}-3 \omega c_{0}-\omega c_{2}-3 \alpha_{4} c_{0}-\alpha_{4} c_{2}=0, \\
& -2 \kappa^{2} \alpha_{1} c_{1}+6 \alpha_{2} c_{0}{ }^{2} c_{1}+\alpha_{2} c_{1}^{3}-6 \alpha_{2} c_{1} c_{2}^{2}+2 D \alpha_{1} c_{1}-8 D \alpha_{3} c_{1} \\
& -6 E \alpha_{1} c_{1}+24 E \alpha_{3} c_{1}+2 F \alpha_{1} c_{1}-8 F \alpha_{3} c_{1}-2 \omega c_{1}-2 \alpha_{4} c_{1}=0, \\
& -3 \kappa^{2} \alpha_{1} c_{0}+\kappa^{2} \alpha_{1} c_{2}+3 \alpha_{2} c_{0}^{3}-3 \alpha_{2} c_{0}{ }^{2} c_{2}+3 \alpha_{2} c_{0} c_{1}^{2} \\
& -3 \alpha_{2} c_{0} c_{2}^{2}-3 \alpha_{2} c_{1}^{2} c_{2}+3 \alpha_{2} c_{2}^{3}+8 E \alpha_{1} c_{2}-32 E \alpha_{3} c_{2} \\
& -12 F \alpha_{1} c_{2}+48 F \alpha_{3} c_{2}-3 \omega c_{0}+\omega c_{2}-3 \alpha_{4} c_{0}+\alpha_{4} c_{2}=0, \\
& -\kappa^{2} \alpha_{1} c_{1}+3 \alpha_{2} c_{0}^{2} c_{1}-6 \alpha_{2} c_{0} c_{1} c_{2}+3 \alpha_{2} c_{1} c_{2}^{2}+E \alpha_{1} c_{1} \\
& -4 E \alpha_{3} c_{1}-6 F \alpha_{1} c_{1}+24 F \alpha_{3} c_{1}-\omega c_{1}-\alpha_{4} c_{1}=0, \\
& -\kappa^{2} \alpha_{1} c_{0}+\kappa^{2} \alpha_{1} c_{2}+\alpha_{2} c_{0}{ }^{3}-3 \alpha_{2} c_{0}^{2} c_{2}+3 \alpha_{2} c_{0} c_{2}^{2}-\alpha_{2} c_{2}^{3} \\
& +4 F \alpha_{1} c_{2}-16 F \alpha_{3} c_{2}-\omega c_{0}+\omega c_{2}-\alpha_{4} c_{0}+\alpha_{4} c_{2}=0 .
\end{aligned}
$$

Solving the above set of non-linear algebraic equations leads to the following cases:

Case 1 . When $D=1, E=-\left(m^{2}+1\right)$, and $F=m^{2}$, we obtain

- $c_{0}=0, \quad c_{1}= \pm \sqrt{-\frac{32 \alpha_{1}-128 \alpha_{3}}{\alpha_{2}}}, \quad c_{2}=0, \omega=-\kappa^{2} \alpha_{1}-8 \alpha_{1}+$ $32 \alpha_{3}-\alpha_{4}, \quad m=1$,

- $c_{0}=0, c_{1}=0, \quad c_{2}= \pm \sqrt{-\frac{-8 \alpha_{1}+32 \alpha_{3}}{\alpha_{2}}}, \omega=-\kappa^{2} \alpha_{1}+4 \alpha_{1}-$ $16 \alpha_{3}-\alpha_{4}, \quad m=1$,

- $c_{0}=0, \quad c_{1}= \pm \sqrt{-\frac{8 \alpha_{1}-32 \alpha_{3}}{\alpha_{2}}}, \quad c_{2}= \pm \sqrt{-\frac{-2 \alpha_{1}+8 \alpha_{3}}{\alpha_{2}}}, \quad \omega=$ $-\kappa^{2} \alpha_{1}-2 \alpha_{1}+8 \alpha_{3}-\alpha_{4}, \quad m=1$,

- $c_{0}=0, \quad c_{1}=\mp \sqrt{-\frac{8 \alpha_{1}-32 \alpha_{3}}{\alpha_{2}}}, \quad c_{2}= \pm \sqrt{-\frac{-2 \alpha_{1}+8 \alpha_{3}}{\alpha_{2}}}, \quad \omega=$ $-\kappa^{2} \alpha_{1}-2 \alpha_{1}+8 \alpha_{3}-\alpha_{4}, \quad m=1$.

Now, the following exact solutions to the CGL equation are retrieved

$$
\begin{aligned}
& u_{1,2}(x, t)= \pm \sqrt{-\frac{32 \alpha_{1}-128 \alpha_{3}}{\alpha_{2}}} \frac{\tanh \left(x+2 \kappa \alpha_{1} t\right)}{1+\tanh ^{2}\left(x+2 \kappa \alpha_{1} t\right)} \\
& \times e^{i\left(-\kappa x+\left(-\kappa^{2} \alpha_{1}-8 \alpha_{1}+32 \alpha_{3}-\alpha_{4}\right) t+\theta\right)}, \\
& u_{3,4}(x, t)= \pm \sqrt{-\frac{-8 \alpha_{1}+32 \alpha_{3}}{\alpha_{2}}} \frac{1-\tanh ^{2}\left(x+2 \kappa \alpha_{1} t\right)}{1+\tanh ^{2}\left(x+2 \kappa \alpha_{1} t\right)}
\end{aligned}
$$

$$
\begin{aligned}
& \times e^{i\left(-\kappa x+\left(-\kappa^{2} \alpha_{1}+4 \alpha_{1}-16 \alpha_{3}-\alpha_{4}\right) t+\theta\right)}, \\
& u_{5,6}(x, t)=\left( \pm \sqrt{-\frac{8 \alpha_{1}-32 \alpha_{3}}{\alpha_{2}}} \frac{\tanh \left(x+2 \kappa \alpha_{1} t\right)}{1+\tanh ^{2}\left(x+2 \kappa \alpha_{1} t\right)}\right. \\
& \pm \sqrt{\left.-\frac{-2 \alpha_{1}+8 \alpha_{3}}{\alpha_{2}} \frac{1-\tanh ^{2}\left(x+2 \kappa \alpha_{1} t\right)}{1+\tanh ^{2}\left(x+2 \kappa \alpha_{1} t\right)}\right)} \\
& \times e^{i\left(-\kappa x+\left(-\kappa^{2} \alpha_{1}-2 \alpha_{1}+8 \alpha_{3}-\alpha_{4}\right) t+\theta\right)}, \\
& u_{7,8}(x, t)=\left(\mp \sqrt{-\frac{8 \alpha_{1}-32 \alpha_{3}}{\alpha_{2}}} \frac{\tanh \left(x+2 \kappa \alpha_{1} t\right)}{1+\tanh \left(x+2 \kappa \alpha_{1} t\right)}\right. \\
& \pm \sqrt{\left.-\frac{-2 \alpha_{1}+8 \alpha_{3}}{\alpha_{2}} \frac{1-\tanh ^{2}\left(x+2 \kappa \alpha_{1} t\right)}{1+\tanh ^{2}\left(x+2 \kappa \alpha_{1} t\right)}\right)} \\
& \times e^{i\left(-\kappa x+\left(-\kappa^{2} \alpha_{1}-2 \alpha_{1}+8 \alpha_{3}-\alpha_{4}\right) t+\theta\right)} .
\end{aligned}
$$

The 3-dimensional graphs of $\left|u_{1}(x, t)\right|$ and $\left|u_{3}(x, t)\right|$ which indicate dark and bright soliton solutions have been shown in Figure 1 for a series of the involved parameters.

Case 2. When $D=1-m^{2}, E=2 m^{2}-1$, and $F=-m^{2}$, we acquire

$$
\begin{aligned}
& c_{0}= \pm \sqrt{-\frac{-\alpha_{1}+4 \alpha_{3}}{2 \alpha_{2}}}, \quad c_{1}=0, \quad c_{2}= \pm \frac{\alpha_{1}-4 \alpha_{3}}{\alpha_{2} \sqrt{-\frac{-\alpha_{1}+4 \alpha_{3}}{2 \alpha_{2}}}} \\
& \omega=-\kappa^{2} \alpha_{1}+\frac{5}{2} \alpha_{1}-10 \alpha_{3}-\alpha_{4}, \quad m=\frac{1}{2}
\end{aligned}
$$

Now, the following Jacobi elliptic function solutions to the CGL equation are derived.

$$
\begin{aligned}
& u_{9,10}(x, t)= \pm \sqrt{-\frac{-\alpha_{1}+4 \alpha_{3}}{2 \alpha_{2}}} \\
& \pm \frac{\alpha_{1}-4 \alpha_{3}}{\alpha_{2} \sqrt{-\frac{-\alpha_{1}+4 \alpha_{3}}{2 \alpha_{2}}}} \frac{1-c n^{2}\left(x+2 \kappa \alpha_{1} t, \frac{1}{2}\right)}{1+c n^{2}\left(x+2 \kappa \alpha_{1} t, \frac{1}{2}\right)} \\
& \times e^{i\left(-\kappa x+\left(-\kappa^{2} \alpha_{1}+\frac{5}{2} \alpha_{1}-10 \alpha_{3}-\alpha_{4}\right) t+\theta\right)} .
\end{aligned}
$$



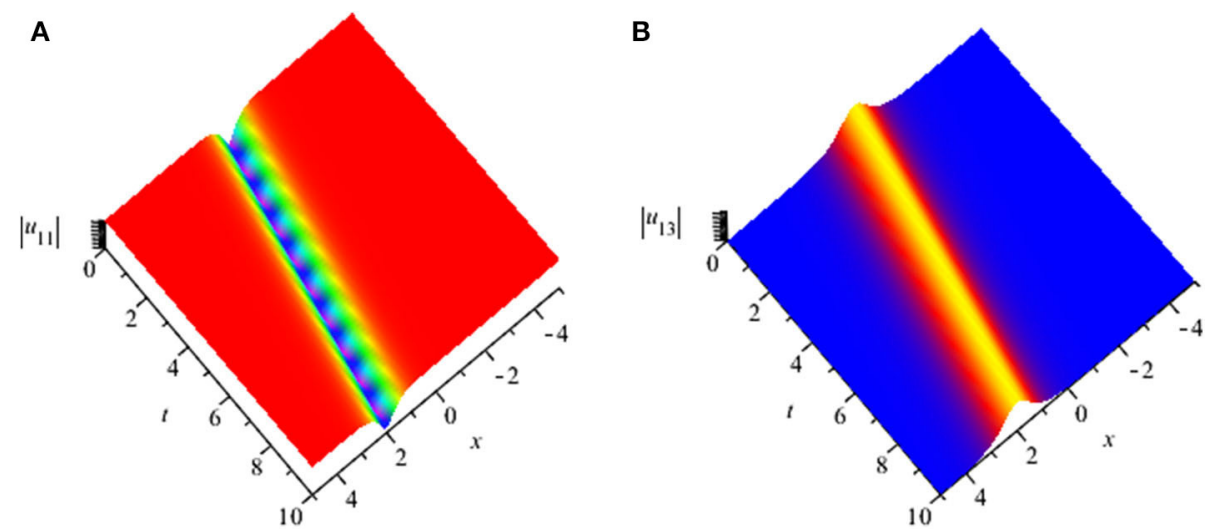

FIGURE 2 | (A) The 3-dimensional graph of $\left|u_{11}(x, t)\right|$ for $\alpha_{1}=1, \alpha_{2}=1, \alpha_{3}=1, \alpha_{4}=1, \kappa=-0.1$, and $\theta=0$; (B) The 3-dimensional graph of $\left|u_{13}(x, t)\right|$ for $\alpha_{1}=1$, $\alpha_{2}=1, \alpha_{3}=1, \alpha_{4}=1, \kappa=-0.1$, and $\theta=0$.

Case 3. When $D=m^{2}, E=-\left(m^{2}+1\right)$, and $F=1$, we obtain

- $c_{0}=0, \quad c_{1}= \pm \sqrt{-\frac{32 \alpha_{1}-128 \alpha_{3}}{\alpha_{2}}}, c_{2}=0, \omega=-\kappa^{2} \alpha_{1}-8 \alpha_{1}+$ $32 \alpha_{3}-\alpha_{4}, \quad m=1$,

- $c_{0}=0, \quad c_{1}=0, \quad c_{2}= \pm \sqrt{-\frac{-8 \alpha_{1}+32 \alpha_{3}}{\alpha_{2}}}, \quad \omega=-\kappa^{2} \alpha_{1}+4 \alpha_{1}-$ $16 \alpha_{3}-\alpha_{4}, \quad m=1$,

- $c_{0}=0, \quad c_{1}= \pm \sqrt{-\frac{8 \alpha_{1}-32 \alpha_{3}}{\alpha_{2}}}, \quad c_{2}= \pm \sqrt{-\frac{-2 \alpha_{1}+8 \alpha_{3}}{\alpha_{2}}}, \quad \omega=$ $-\kappa^{2} \alpha_{1}-2 \alpha_{1}+8 \alpha_{3}-\alpha_{4}, \quad m=1$,

- $c_{0}=0, \quad c_{1}=\mp \sqrt{-\frac{8 \alpha_{1}-32 \alpha_{3}}{\alpha_{2}}}, \quad c_{2}= \pm \sqrt{-\frac{-2 \alpha_{1}+8 \alpha_{3}}{\alpha_{2}}}, \quad \omega=$ $-\kappa^{2} \alpha_{1}-2 \alpha_{1}+8 \alpha_{3}-\alpha_{4}, \quad m=1$.

Now, the following exact solutions to the CGL equation are retrieved

$$
\begin{aligned}
& u_{11,12}(x, t)= \pm \sqrt{-\frac{32 \alpha_{1}-128 \alpha_{3}}{\alpha_{2}}} \frac{\operatorname{coth}\left(x+2 \kappa \alpha_{1} t\right)}{1+\operatorname{coth}^{2}\left(x+2 \kappa \alpha_{1} t\right)} \\
& \times e^{i\left(-\kappa x+\left(-\kappa^{2} \alpha_{1}-8 \alpha_{1}+32 \alpha_{3}-\alpha_{4}\right) t+\theta\right)}, \\
& u_{13,14}(x, t)= \pm \sqrt{-\frac{-8 \alpha_{1}+32 \alpha_{3}}{\alpha_{2}}} \frac{1-\operatorname{coth}^{2}\left(x+2 \kappa \alpha_{1} t\right)}{1+\operatorname{coth}^{2}\left(x+2 \kappa \alpha_{1} t\right)} \\
& \times e^{i\left(-\kappa x+\left(-\kappa^{2} \alpha_{1}+4 \alpha_{1}-16 \alpha_{3}-\alpha_{4}\right) t+\theta\right)}, \\
& u_{15,16}(x, t)=\left( \pm \sqrt{-\frac{8 \alpha_{1}-32 \alpha_{3}}{\alpha_{2}}} \frac{\operatorname{coth}\left(x+2 \kappa \alpha_{1} t\right)}{1+\operatorname{coth}\left(x+2 \kappa \alpha_{1} t\right)}\right. \\
& \pm \sqrt{\left.-\frac{-2 \alpha_{1}+8 \alpha_{3}}{\alpha_{2}} \frac{1-\operatorname{coth}^{2}\left(x+2 \kappa \alpha_{1} t\right)}{1+\operatorname{coth}^{2}\left(x+2 \kappa \alpha_{1} t\right)}\right)} \\
& \times e^{i\left(-\kappa x+\left(-\kappa^{2} \alpha_{1}-2 \alpha_{1}+8 \alpha_{3}-\alpha_{4}\right) t+\theta\right)}, \\
& u_{17,18}(x, t)=\left(\mp \sqrt{-\frac{8 \alpha_{1}-32 \alpha_{3}}{\alpha_{2}}} \frac{\operatorname{coth}^{2}\left(x+2 \kappa \alpha_{1} t\right)}{1+\operatorname{coth}^{2}\left(x+2 \kappa \alpha_{1} t\right)}\right. \\
& \left. \pm \sqrt{-\frac{-2 \alpha_{1}+8 \alpha_{3}}{\alpha_{2}}} \frac{1-\operatorname{coth}^{2}\left(x+2 \kappa \alpha_{1} t\right)}{1+\operatorname{coth}^{2}\left(x+2 \kappa \alpha_{1} t\right)}\right) \\
& \times e^{i\left(-\kappa x+\left(-\kappa^{2} \alpha_{1}-2 \alpha_{1}+8 \alpha_{3}-\alpha_{4}\right) t+\theta\right)} .
\end{aligned}
$$

The 3-dimensional graphs of $\left|u_{11}(x, t)\right|$ and $\left|u_{13}(x, t)\right|$ which denote dark and bright soliton solutions have been presented in Figure 2 for a series of the involved parameters.

Case 4 . When $D=-m^{2}, E=2 m^{2}-1$, and $F=1-m^{2}$, we acquire

$$
\begin{aligned}
& c_{0}= \pm \sqrt{-\frac{-\alpha_{1}+4 \alpha_{3}}{2 \alpha_{2}}}, \quad c_{1}=0, \quad c_{2}=\mp \frac{\alpha_{1}-4 \alpha_{3}}{\alpha_{2} \sqrt{-\frac{-\alpha_{1}+4 \alpha_{3}}{2 \alpha_{2}}}} \\
& \omega=-\kappa^{2} \alpha_{1}+\frac{5}{2} \alpha_{1}-10 \alpha_{3}-\alpha_{4}, \quad m=\frac{1}{2}
\end{aligned}
$$

Now, the following Jacobi elliptic function solutions to the CGL equation are derived.

$$
\begin{aligned}
& u_{19,20}(x, t)= \pm \sqrt{-\frac{-\alpha_{1}+4 \alpha_{3}}{2 \alpha_{2}}} \\
& \mp \frac{\alpha_{1}-4 \alpha_{3}}{\alpha_{2} \sqrt{-\frac{-\alpha_{1}+4 \alpha_{3}}{2 \alpha_{2}}}} \frac{1-n c^{2}\left(x+2 \kappa \alpha_{1} t, \frac{1}{2}\right)}{1+n c^{2}\left(x+2 \kappa \alpha_{1} t, \frac{1}{2}\right)} \\
& \times e^{i\left(-\kappa x+\left(-\kappa^{2} \alpha_{1}+\frac{5}{2} \alpha_{1}-10 \alpha_{3}-\alpha_{4}\right) t+\theta\right)} .
\end{aligned}
$$

\section{CONCLUSION}

The complex Ginzburg-Landau equation as a famous NLPD equation was studied successfully in the current paper. A modified version of the Jacobi elliptic expansion method was formally adopted to carry out this goal. A wide variety of exact solutions to the CGL equation in the presence of the detuning factor were derived. Particularly, several dark and bright soliton solutions were derived when the modulus of ellipticity approaches unity. Although, the exact solutions given in the present paper provide useful information about the complex Ginzburg-Landau equation, seeking other exact solutions of the CGL equation can be considered as an important task in our future works. 


\section{DATA AVAILABILITY STATEMENT}

The original contributions presented in the study are included in the article/supplementary material, further inquiries can be directed to the corresponding author/s.

\section{AUTHOR CONTRIBUTIONS}

$\mathrm{KH}$ and DB: conceptualization, project administration, and Supervision. KH: data curation and visualization. $\mathrm{KH}$ and

\section{REFERENCES}

1. Biswas A, Ekici M, Triki H, Sonmezoglu A, Mirzazadeh M, Zhou Q, et al. Resonant optical soliton perturbation with anti-cubic nonlinearity by extended trial function method. Optik. (2018) 156:78490. doi: 10.1016/j.ijleo.2017.12.035

2. Biswas A, Ekici M, Sonmezoglu A, Alqahtani RT. Sub-picosecond chirped optical solitons in mono-mode fibers with KaupNewell equation by extended trial function method. Optik. (2018) 168:208-16. doi: 10.1016/j.ijleo.2018.04.069

3. Lu D, Seadawy A, Arshad M. Applications of extended simple equation method on unstable non-linear Schr?dinger equations. Optik. (2017) 140:13644. doi: 10.1016/j.ijleo.2017.04.032

4. Arnous AH, Ullah MZ, Moshokoa SP, Zhou Q, Triki H, Mirzazadeh M, et al. Optical solitons in birefringent fibers with modified simple equation method. Optik. (2017) 130:996-1003. doi: 10.1016/j.ijleo.2016.11.101

5. Kudryashov NA. One method for finding exact solutions of non-linear differential equations. Commun Non-Linear Sci Numerical Simulations. (2012) 17:2248-53. doi: 10.1016/j.cnsns.2011.10.016

6. Hosseini K, Korkmaz A, Bekir A, Samadani F, Zabihi A, Topsakal M. New wave form solutions of non-linear conformable time-fractional Zoomeron equation in (2+1)-dimensions. Waves Random Complex Media. (2019) (in press). doi: 10.1080/17455030.2019.1579393

7. Hosseini K, Yazdani Bejarbaneh E, Bekir A, Kaplan M. New exact solutions of some non-linear evolution equations of pseudoparabolic type. Optical Quantum Electr. (2017) 49:241. doi: 10.1007/s11082-017-1070-z

8. Hosseini K, Mayeli P, Kumar D. New exact solutions of the coupled sineGordon equations in non-linear optics using the modified Kudryashov method. J Modern Opt. (2018) 65:361-4. doi: 10.1080/09500340.2017.1380857

9. Yan C. A simple transformation for non-linear waves. Phys Lett A. (1996) 224:77-84. doi: 10.1016/S0375-9601(96)00770-0

10. Kumar D, Hosseini K, Samadani F. The sine-Gordon expansion method to look for the traveling wave solutions of the Tzitzéica type equations in non-linear optics. Optik. (2017) 149:439-46. doi: 10.1016/j.ijleo.2017.09.066

11. Ali KK, Wazwaz AM, Osman MS. Optical soliton solutions to the generalized non-autonomous non-linear Schrödinger equations in optical fibers via the sine-Gordon expansion method. Optik. (2019) 204:164132. doi: 10.1016/j.ijleo.2019.164132

12. Ali KK, Osman MS, Abdel-Aty M. New optical solitary wave solutions of Fokas-Lenells equation in optical fiber via Sine-Gordon expansion method. Alexandria Eng J. (2020) 59:1191-96. doi: 10.1016/j.aej.2020.01.037

13. Hosseini K, Mirzazadeh M, Ilie M, Radmehr S. Dynamics of optical solitons in the perturbed Gerdjikov-Ivanov equation. Optik. (2020) 206:164350. doi: 10.1016/j.ijleo.2020.164350

14. Hosseini K, Osman MS, Mirzazadeh M, Rabiei F. Investigation of different wave structures to the generalized third-order non-linear Scrödinger equation. Optik. (2020) 206:164259. doi: 10.1016/j.ijleo.2020.164259

15. Hosseini K, Mirzazadeh M, Zhou Q, Liu Y, Moradi M. Analytic study on chirped optical solitons in non-linear metamaterials with higher order effects. Laser Phys. (2019) 29:095402. doi: 10.1088/1555-6611/ab356f

16. Liu JG, Osman MS, Zhu WH, Zhou L, Ai GP. Different complex wave structures described by the Hirota equation with variable
MM: formal analysis, investigation, and writing-original draft. MA and DB: funding acquisition. $\mathrm{KH}, \mathrm{MM}, \mathrm{MO}$, and MA: methodology, software, and validation. $\mathrm{KH}, \mathrm{MM}$, $\mathrm{MO}$, MA, and DB: resources. $\mathrm{KH}, \mathrm{MM}$, and MO: writingreview editing. All persons who meet authorship criteria are listed as authors, and all authors certify that they have participated sufficiently in the work to take public responsibility for the content, including participation in the concept, design, analysis, writing, or revision of the manuscript.

coefficients in inhomogeneous optical fibers. Appl Phys B. (2019) 125:175. doi: 10.1007/s00340-019-7287-8

17. Ghanbari B, Osman MS, Baleanu D. Generalized exponential rational function method for extended Zakharov-Kuzetsov equation with conformable derivative. Modern Phys Lett A. (2019) 34:1950155. doi: 10.1142/S0217732319501554

18. Lu D, Tariq KU, Osman MS, Baleanu D, Younis M, Khater MMA. New analytical wave structures for the (3+1)-dimensional Kadomtsev-Petviashvili and the generalized Boussinesq models and their applications. Results Phys. (2019) 14:102491. doi: 10.1016/j.rinp.2019.102491

19. Shang Y. Lie algebraic discussion for affinity based information diffusion in social networks, Acta Physica Polonica B. (2015) 46:1567-77. doi: 10.5506/APhysPolB.46.1567

20. Shang Y. Analytical solution for an in-host viral infection model with time-inhomogeneous rates. Open Phys. (2017) 15:705-11. doi: 10.1515/phys-2017-0083

21. Ma HC, Zhang ZP, Deng AP. A new periodic solution to Jacobi elliptic functions of $\mathrm{MKdV}$ equation and BBM equation. Acta Mathematicae Applicatae Sinica. (2012) 28:409-15. doi: 10.1007/s10255-012-0153-7

22. Zayed EME, Shohib RMA, Biswas A, Yildirim Y, Mallawi F, Belic MR. Chirped and chirp-free solitons in optical fiber Bragg gratings with dispersive reflectivity having parabolic law non-linearity by Jacobi's elliptic function. Results Phys. (2019) 15:102784. doi: 10.1016/j.rinp.2019.102784

23. Zayed EME, Alngar MEM. Optical solitons in birefringent fibers with BiswasArshed model by generalized Jacobi elliptic function expansion method. Optik. (2020) 203:163922. doi: 10.1016/j.ijleo.2019.163922

24. Hosseini K, Mirzazadeh M, Ilie M, Gómez-Aguilar JF. Biswas-Arshed equation with the beta time derivative: optical solitons and other solutions. Optik. (2020) 217:164801. doi: 10.1016/j.ijleo.2020.164801

25. El-Sheikh MMA, Seadawy AR, Ahmed HM, Arnous AH, Rabie WB. Dispersive and propagation of shallow water waves as a higher order non-linear Boussinesq-like dynamical wave equations. Physica A. (2020) 537:122662. doi: 10.1016/j.physa.2019.122662

26. Hosseini K, Mirzazadeh M, Vahidi J, Asghari R. Optical wave structures to the Fokas-Lenells equation. Optik. (2020) 207:164450. doi: 10.1016/j.ijleo.2020.164450

27. Hosseini K, Matinfar M, Mirzazadeh M. A (3+1)-dimensional resonant nonlinear Schrödinger equation and its Jacobi elliptic and exponential function solutions. Optik. (2020) 207:164458. doi: 10.1016/j.ijleo.2020.164458

28. Mirzazadeh M, Ekici M, Sonmezoglu A, Eslami M, Zhou Q. Kara AH, et al. Optical solitons with complex Ginzburg-Landau equation. Non-linear Dyn. (2016) 85:1979-2016. doi: 10.1007/s11071-016-2810-5

29. Osman MS, Ghanbari B, Machado JAT. New complex waves in non-linear optics based on the complex Ginzburg-Landau equation with Kerr law non-linearity. Eur Phys J Plus. (2019) 134:20. doi: 10.1140/epjp/i2019-12442-4

30. Osman MS, Lu D, Khater MMA, Attia RAM. Complex wave structures for abundant solutions related to the complex GinzburgLandau model. Optik. (2019) 192:162927. doi: 10.1016/j.ijleo.2019. 06.027

31. Abdou MA, Soliman AA, Biswas A, Ekici M, Zhou Q, Moshokoa SP. Darksingular combo optical solitons with fractional complex Ginzburg-Landau equation. Optik. (2018) 171:463-7. doi: 10.1016/j.ijleo.2018.06.076 
32. Arshed S. Soliton solutions of fractional complex Ginzburg-Landau equation with Kerr law and non-Kerr law media. Optik. (2018) 160:32232. doi: 10.1016/j.ijleo.2018.02.022

33. Rezazadeh H. New solitons solutions of the complex GinzburgLandau equation with Kerr law non-linearity. Optik. (2018) 167:218-27. doi: 10.1016/j.ijleo.2018.04.026

34. Arnous AH, Seadawy AR, Alqahtani RT, Biswas A. Optical solitons with complex Ginzburg-Landau equation by modified simple equation method. Optik. (2017) 144:475-80. doi: 10.1016/j.ijleo.2017.07.013

35. Hancock H. Lectures on the Theory of Elliptic Functions. New York, NY: J. Wiley \& Sons (1910)
Conflict of Interest: The authors declare that the research was conducted in the absence of any commercial or financial relationships that could be construed as a potential conflict of interest.

Copyright (C) 2020 Hosseini, Mirzazadeh, Osman, Al Qurashi and Baleanu. This is an open-access article distributed under the terms of the Creative Commons Attribution License (CC BY). The use, distribution or reproduction in other forums is permitted, provided the original author(s) and the copyright owner(s) are credited and that the original publication in this journal is cited, in accordance with accepted academic practice. No use, distribution or reproduction is permitted which does not comply with these terms. 